Asia Pac. J. Math. 2019 6:16

\title{
NEW EXACT AND NUMERICAL SOLUTIONS OF FRACTIONAL KAUP-KUPERSHMIDT EQUATION
}

\author{
MEHMET SENOL ${ }^{1}$, ORKUN TASBOZAN $^{2}$, ALI KURT $^{3, *}$, AYŞE ATA $^{1}$ \\ ${ }^{1}$ Department of Mathematics, Faculty of Science and Art, Nevsehir Haci Bektas Veli University, Nevsehir, TURKEY \\ ${ }^{2}$ Department of Mathematics, Faculty of Science and Art, Hatay Mustafa Kemal University, Hatay, TURKEY \\ ${ }^{3}$ Department of Mathematics, Faculty of Science and Art, Pamukkale University, Denizli, TURKEY \\ *Corresponding Author: pau.dr.alikurt@gmail.com, akurt@pau.edu.tr \\ Received May 31, 2019
}

\begin{abstract}
AвSTRACT. In this article, the tanh method and the residual power series method (RPSM) are used to obtain new exact and numerical solutions of the time-fractional Kaup-Kupershmidt equation using the conformable fractional derivative definition. This definition is simple, effective and reliable in the solution procedure of the fractional differential equations that have complicated solutions with classical fractional derivative definitions like Caputo and Rieman-Liouville. 2010 Mathematics Subject Classification. 35R11; 35A20; 35C05.
\end{abstract}

Key words and phrases. Conformable Fractional Derivative, Fractional Kaup-Kupershmidt Equation, Tanh Method, Residual Power Series Method.

\section{InTRODUCTION}

Fractional calculus has found numerous applications in science and engineering branches such as fractional differential equations (FDE), fluid flow, electrical network, mathematical physics, biology, image and signal processing, viscoelasticity and control in recent years.

There are some common methods that are used to obtain approximate or analytical solutions of nonlinear fractional partial differential equations in literature. Adomian decomposition method (ADM) [19], Laplace analysis method (LAM) [13], homotopy analysis method (HAM) [16], homotopy perturbation method (HPM) [22], differential transformation method (DTM) [5] and perturbation-iteration algoritm (PIA) [20] are among them.

In this article, the tanh method $[8,21]$ and residual power series method (RPSM) $[2,4,11,12,15]$ are used to obtain new exact and approximate solutions of time-fractional Kaup-Kumershmidt equation of the form

$$
\frac{\partial^{\alpha} u}{\partial t^{\alpha}}+45 u^{2} \frac{\partial u}{\partial x}-15 p \frac{\partial u}{\partial x} \frac{\partial^{2} u}{\partial x^{2}}-15 u \frac{\partial^{3} u}{\partial x^{3}}+\frac{\partial^{5} u}{\partial x^{5}}=0
$$

The tanh method is a powerful tool for obtaining traveling wave solutions of nonlinear fractional differential equations. In this method a power series in tanh was used as an ansatz to obtain analytical solutions of traveling

DOI: 10.28924/APJM/6-16

(92019 Asia Pacific Journal of Mathematics 
wave type of certain evolution equations. Besides, in RPSM, the coefficients of the power series are calculated by means of the concept of residual error with the help of one or more variable algebraic equation chains, and finally, a so-called truncated series solution is obtained [15].

The major improvement of the RPSM is that it can be implemented to the problem directly without linearization, perturbation or discretization and without any transformation by selecting appropriate initial conditions [12].

After giving brief descriptions of tanh method and RSPM, we have presented one example that shows reliability and efficiency of two methods. Also figures and a table are presented in order to compare their numerical results. At last, we discussed about obtained results as a section for conclusion.

\section{Preliminaries}

There are a few definition of fractional derivative of order $\alpha>0$. The most widely used are the RiemannLiouville and Caputo fractional derivatives.

Definition 2.1. The Riemann-Liouville fractional derivative operator $D^{\alpha} f(x)$ for $\alpha>0$ and $q-1<\alpha<q$ defined as $[2,9,10]$ :

$$
D^{\alpha} f(x)=\frac{d^{q}}{d x^{q}}\left[\frac{1}{\Gamma(q-\alpha)} \int_{\alpha}^{x} \frac{f(t)}{(x-t)^{\alpha+1-q}} d t\right]
$$

Definition 2.2. The Caputo fractional derivative of order $\alpha>0$ for $n \in \mathbb{N}, n-1<\alpha<n, D_{*}^{\alpha}$, defined as [7]:

$$
D_{*}^{\alpha} f(x)=J^{n-\alpha} D^{n} f(x)=\frac{1}{\Gamma(n-\alpha)} \int_{\alpha}^{x}(x-t)^{n-\alpha-1}\left(\frac{d}{d t}\right)^{n} f(t) d t
$$

Recently, a new definition of a fractional derivative called the "conformable fractional derivative" has been proposed by R. Khalil et al. [6].

Definition 2.3. Let $f:[0, \infty) \rightarrow R$ is a function $\alpha$ - th order "conformable fractional derivative" of a defined by

$$
T_{\alpha}(f)(t)=\lim _{\varepsilon \rightarrow 0} \frac{f\left(t+\varepsilon t^{1-\alpha}\right)-(f)(t)}{\varepsilon}
$$

for all $t>0, \alpha \in(0,1)$.

The properties of this new definition are given in the following theorem [14]

Theorem 2.1. Let $\alpha \in(0,1]$ and $f, g$ functions are $\alpha$-differantiable at point $t>0$, then

1. $T_{\alpha}(m f+n g)=m T_{\alpha}(f)+n T_{\alpha}(g)$ for all $m, n \in \mathbb{R}$

2. $T_{\alpha}\left(t^{p}\right)=p t^{p-\alpha}$ for all $p$

3. $T_{\alpha}(f . g)=f T_{\alpha}(g)+g T_{\alpha}(f)$

4. $T_{\alpha}\left(\frac{f}{g}\right)=\frac{g T_{\alpha}(f)-f T_{\alpha}(g)}{g^{2}}$

5. $T_{\alpha}(c)=0$ for all constant functions $f(t)=c$

6. If, in addition, $\mathrm{f}$ is differentiable, then $T_{\alpha}(f)(t)=t^{1-\alpha} \frac{d f(t)}{d t}$ 
Definition 2.4. Let $f$ is a function with $n$ variables $x_{1}, \ldots, x_{n}$, and the conformable partial derivatives of $f$ of order $\alpha \in(0,1]$ in $x_{i}$ is defined as follows [6]

$$
\frac{d^{\alpha}}{d x_{i}^{\alpha}} f\left(x_{1}, \ldots, x_{n}\right)=\lim _{\varepsilon \rightarrow 0} \frac{f\left(x_{1}, \ldots, x_{i-1}, x_{i}+\varepsilon x_{i}^{1-\alpha}, \ldots, x_{n}\right)-f\left(x_{1}, \ldots, x_{n}\right)}{\varepsilon} .
$$

Definition 2.5. The conformable integral of a function $f$ starting from $a \geq 0$ is defined as [16]

$$
I_{\alpha}^{a}(f)(s)=\int_{a}^{s} \frac{f(t)}{t^{1-\alpha}} d t .
$$

\section{A Brief Description of Implemented Methods}

\subsection{The Tanh Method.}

In the beginning we are going to describe the method [21] incrementally.

Step1. The general form of nonlinear conformable fractional differential equation can be regarded as

$$
P\left(\frac{\partial^{\alpha} u}{\partial t^{\alpha}}, \frac{\partial u}{\partial x}, \frac{\partial^{2 \alpha} u}{\partial t^{2 \alpha}}, \frac{\partial^{2} u}{\partial x^{2}}, \ldots\right)=0
$$

where the arguments and subscripts of polynomial $P$ shows partial derivatives.

Step2. Employing the transformation

$$
u(x, t)=u(\xi), \xi=k x-m \frac{t^{\alpha}}{\alpha}
$$

in which $k$ denotes the number of wave and $m$ shows the velocity of the wave. Due to this:

$$
\frac{\partial^{\alpha}(.)}{\partial t^{\alpha}}=n \frac{d(.)}{d \xi}, \frac{\partial(.)}{\partial x}=m \frac{d(.)}{d \xi}, \ldots
$$

Considering Eq.(3.2), Eq.(3.1) turns in to a differential equation

$$
G\left(U, U^{\prime}, U^{\prime \prime}, U^{\prime \prime \prime}, \ldots\right)=0
$$

where the derivatives are with respect to $\xi$.

Step3. Now, describing a new independent variable

$$
Y=\tanh (\xi)
$$

Then the following equations are hold.

$$
\begin{gathered}
\frac{\partial}{\partial \xi}=\left(1-Y^{2}\right) \frac{\partial}{\partial Y} \\
\frac{\partial^{2}}{\partial \xi^{2}}=-2 Y\left(1-Y^{2}\right) \frac{\partial}{\partial Y}+\left(1-Y^{2}\right)^{2} \frac{\partial^{2}}{\partial Y^{2}}, \\
\frac{\partial^{3}}{\partial \xi^{3}}=2(1-Y)^{2}\left(3 Y^{2}-1\right) \frac{\partial}{\partial Y}-6 Y\left(1-Y^{2}\right)^{2} \frac{\partial^{2}}{\partial Y^{2}}+\left(\left(1-Y^{2}\right)^{3}\right) \frac{\partial^{3}}{\partial Y^{3}}, \\
\frac{\partial^{4}}{\partial \xi^{4}}=-8 Y\left(1-Y^{2}\right)\left(3 Y^{2}-2\right) \frac{\partial}{\partial Y}+4\left(1-Y^{2}\right)^{2}\left(9 Y^{2}-2\right) \frac{\partial^{2}}{\partial Y^{2}}-12 Y\left(1-Y^{2}\right)^{3} \frac{\partial^{3}}{\partial Y^{3}}+\left(1-Y^{2}\right)^{4} \frac{\partial^{4}}{\partial Y^{4}},
\end{gathered}
$$




$$
\begin{aligned}
& \frac{\partial^{5}}{\partial \xi^{5}}=-8 Y\left(-1+Y^{2}\right)\left(2-15 Y^{2}+15 Y^{4}\right) \frac{\partial}{\partial Y}-120 Y\left(-1+Y^{2}\right)^{2}\left(-1+2 Y^{2}\right) \frac{\partial^{2}}{\partial Y^{2}} \\
& -20\left(-1+Y^{2}\right)^{3}\left(-1+6 Y^{2}\right) \frac{\partial^{3}}{\partial Y^{3}}-20 Y\left(-1+Y^{2}\right)^{4} \frac{\partial^{4}}{\partial Y^{4}}+\left(1-Y^{2}\right)^{5} \frac{\partial^{5}}{\partial Y^{5}} .
\end{aligned}
$$

Step4. Present the prediction

$$
U(\xi)=S(Y)=\sum_{i=0}^{r} a_{i} Y^{i}
$$

where $r$ is a positive integer, in most cases, that will be evaluated. Substituting (3.7) into the ODE (3.4) concludes an equation in powers of $Y$.

Step5. Balancing the linear terms of highest order in the ODE (3.4) with the highest order nonlinear terms gives us the parameter $r$. After obtaining $r$, the all coefficients of $Y$ are equated to zero in the resulting equation. This results a system of algebraic equations including $k, m, p$ and $a_{i},(i=0,1, \ldots, r)$. The analytical solution is obtained in a closed form after having determined these parameters, considering that $r$ is a positive integer, and using (3.7).

To be very competent on the the tanh method, one can look into Ref. $[17,18]$ for the analysis with useful discussions.

\subsection{Description of the residual power series method.}

In this section we are going to introduce some important definitions and theorems about residual power series

Theorem 3.1. Suppose that $f$ has a FPS representation at $t_{0}=0$ of the form

$$
f(t)=\sum_{n=0}^{\infty} c_{n} t^{n \alpha}, 0<t<R^{\frac{1}{\alpha}}, R>0
$$

where $R^{\frac{1}{\alpha}}$ is the radius of convergence. If $f$ is an infinitely conformable $\alpha$-differentiable function, for some $0 \leq m-1<$ $\alpha \leq m$ in a neigborhood of a point $t_{0}=0$, then the coefficients $c_{n}$ in $(3.8)$ will take the form $c_{n}=\frac{f^{(n \alpha)}(0)}{\alpha^{n} n !}$ where $f^{(n \alpha)}(t)$ means the application of the conformable fractional derivative $n$ times [1].

Definition 3.1. A multiple fractional power series about $t_{0}=0$ is defined by $\sum_{n=0}^{\infty} f_{n}(x) t^{\alpha}$, where $f_{n}(x)$ are the coefficients of the series depend on $x$ and $t$ is a variable.

Definition 3.2. A power series of the form $\sum_{n=0}^{\infty} f_{n}(x) t^{\alpha}$, is called a multiple fractional power series about $t_{0}=0$, where $t$ is a variable and $f_{n}(x)$ are functions of $x$ called the coefficients of the series [11].

Theorem 3.2. Assume that $u(x, t)$ has a multiple fractional power series representation at $t_{0}=0$ of the form [3]

$$
u(x, t)=\sum_{n=0}^{\infty} f_{n}(x) t^{n \alpha}, 0 \leq m-1<\alpha<m, x \in I, 0 \leq t \leq R^{\frac{1}{\alpha}} .
$$

If $u_{t}^{(n \alpha)}(x, t), n=0,1,2, \ldots$ are continuous on $I \times\left(0, R^{\frac{1}{\alpha}}\right)$, then $f_{n}(x)=\frac{u_{t}^{(n \alpha)}(x, 0)}{\alpha^{n} n !}$.

To clarify the basic concept of RPSM, let's take a nonlinear fractional differential equation of the form:

$$
T_{\alpha} u(x, t)+N[x] u(x, t)+R[x] u(x, t)=c(x, t), x \in \mathbb{R}, n-1<n \alpha \leq n, t>0
$$


expressed by initial condition

$$
f_{0}(x)=u(x, 0)=f(x)
$$

where $R[x]$ is a linear, $N[x]$ is a non-linear operator and $c(x, t)$ are continuous functions.

The RPSM method made up of stating the solution of the equation (3.10) subject to (3.11) as a fractional power series expansion around $t=0$.

$$
f_{(n-1)}(x)=T_{t}^{(n-1) \alpha} u(x, 0)=h(x)
$$

The expansion form of the solution is given by

$$
u(x, t)=f(x)+\sum_{n=0}^{\infty} f_{n}(x) \frac{t^{n \alpha}}{\alpha^{n} n !}
$$

In the next step, the $k$.truncted series of $u(x, t)$, namely $u_{k}(x, t)$ can be written as:

$$
u_{k}(x, t)=f(x)+\sum_{n=0}^{k} f_{n}(x) \frac{t^{n \alpha}}{\alpha^{n} n !}
$$

If the 1. RPS approximate solution $u_{1}(x, t)$ is

$$
u_{1}(x, t)=f(x)+f_{1}(x) \frac{t^{\alpha}}{\alpha^{n}}
$$

then $u_{k}(x, t)$ could be reformulated as

$$
u_{k}(x, t)=f(x)+f_{1}(x) \frac{t^{\alpha}}{\alpha^{n}}+\sum_{n=2}^{k} f_{n}(x) \frac{t^{n \alpha}}{\alpha^{n} n !}
$$

for $0<\alpha \leq 1,0 \leq t<\mathbb{R}^{\frac{1}{v}}, x \in I$ and $k=2,3,4, \ldots$

First we express the residual function as

$$
\operatorname{Res}(x, t)=T_{\alpha} u(x, t)+N[x] u(x, t)+R[x] u(x, t)-c(x, t)
$$

and the $k$. residual function as

$$
\operatorname{Res}_{k}(x, t)=T_{\alpha} u_{k}(x, t)+N[x] u_{k}(x, t)+R[x] u_{k}(x, t)-g(x, t), k=1,2,3, \ldots
$$

It is clear that $\operatorname{Res}(x, t)=0$ and $\lim _{k \rightarrow \infty} \operatorname{Res}_{k}(x, t)=\operatorname{Res}(x, t)$ for each $x \in I$ and $0 \leq t$. In fact this lead to $\frac{\partial^{(n-1) \alpha}}{\partial t^{(n-1) \alpha}} \operatorname{Res}_{k}(x, t)$ for $n=1,2,3, \ldots, k$ because in the conformable sense, the fractional derivative of a constant is zero $[4,12,15]$. Solving the equation $\frac{\partial^{(n-1) \alpha}}{\partial t^{(n-1) \alpha}} \operatorname{Res}_{k}(x, 0)=0$ gives us the desired $f_{n}(x)$ coefficients. Thus the $u_{n}(x, t)$ approximate solutions can be obtained respectively. 


\section{Application of Methods for Solving Fractional Kaup-Kupershmidt Equation}

\subsection{Application of Tanh Method.}

Regard the conformable time-fractional Kaup-Kupershmidt equation

$$
\frac{\partial^{\alpha} u}{\partial t^{\alpha}}+45 u^{2} \frac{\partial u}{\partial x}-15 p \frac{\partial u}{\partial x} \frac{\partial^{2} u}{\partial x^{2}}-15 u \frac{\partial^{3} u}{\partial x^{3}}+\frac{\partial^{5} u}{\partial x^{5}}=0
$$

where $\alpha \in(0,1)$. Using the wave transform (3.2) and (3.3), the equation (4.1) becomes

$$
-m u^{\prime}+45 k u^{2} u^{\prime}-15 k^{3} p u^{\prime} u^{\prime \prime}-15 k^{3} u^{\prime} u^{\prime \prime \prime}+k^{5} u^{(5)}
$$

where the prime symbolizes the derivation with respect to $\xi$. Then using the ansatz

$$
Y=\tanh (\xi)
$$

and

$$
u=S(Y)=\sum_{i=0}^{r} a_{i} Y^{i}
$$

When we balance the highest order linear terms in the resulting equation with the highest order nonlinear terms in Eq.(4.2), we obtain $r=2$ and using (4.4) and (3.6) in resulting equation we get

$$
\begin{aligned}
& -\quad m\left(1-Y^{2}\right) \frac{d S}{d Y}+45 k S^{2}\left(1-Y^{2}\right) \frac{d S}{d Y}-15 k^{3} p\left(1-Y^{2}\right) \frac{d S}{d Y}\left(-2 Y\left(1-Y^{2}\right) \frac{d S}{d Y}+\left(1-Y^{2}\right)^{2} \frac{d^{2} S}{d Y^{2}}\right) \\
& -\quad 15 k^{3} S\left(2\left(1-Y^{2}\right)\left(3 Y^{2}-1\right) \frac{d S}{d Y}-6 Y\left(1-Y^{2}\right)^{2} \frac{d^{2} S}{d Y^{2}}\right)+k^{5}\left(-8\left(-1+Y^{2}\right)\left(2-15 Y^{2}+15 Y^{4}\right) \frac{d S}{d Y}\right. \\
& \left.-\quad 120 Y\left(-1+Y^{2}\right)^{2}\left(-1+2 Y^{2}\right) \frac{d^{2} S}{d Y^{2}}\right)=0 .
\end{aligned}
$$

Using obtained values in (4.4), subrogating into (4.5), equating all coefficients of $Y$ led to an algebraic equation system for $a_{0}, a_{1}, a_{2}, k, m$ as follows.

$$
\begin{aligned}
& 45 a_{0}^{2} a_{1} k+30 a_{0} a_{1} k^{3}+16 a_{1} k^{5}-a_{1} m-30 a_{1} a_{2} k^{3} p=0, \\
& 90 a_{0} a_{1}^{2} k+90 a_{0}^{2} a_{2} k+30 a_{1}^{2} k^{3}+240 a_{0} a_{2} k^{3}+272 a_{2} k^{5}-2 a_{2} m+30 a_{1}^{2} k^{3} p-60 a_{2}^{2} k^{3} p=0, \\
& -45 a_{0}^{2} a_{1} k+45 a_{1}^{3} k+270 a_{0} a_{1} a_{2} k-120 a_{0} a_{1} k^{3}+270 a_{1} a_{2} k^{3}-136 a_{1} k^{5}+a_{1} m+210 a_{1} a_{2} k^{3} p=0, \\
& -90 a_{0} a_{1}^{2} k-90 a_{0}^{2} a_{2} k+180 a_{1}^{2} a_{2} k+180 a_{0} a_{2}^{2} k-120 a_{1}^{2} k^{3}-600 a_{0} a_{2} k^{3}+240 a_{2}^{2} k^{3} \\
& -1232 a_{2} k^{5}+2 a_{2} m-60 a_{1}^{2} k^{3} p+300 a_{2}^{2} k^{3} p=0, \\
& -45 a_{1}^{3} k-270 a_{0} a_{1} a_{2} k+225 a_{1} a_{2}^{2} k+90 a_{0} a_{1} k^{3}-720 a_{1} a_{2} k^{3}+240 a_{1} k^{5}-330 a_{1} a_{2} k^{3} p=0, \\
& -180 a_{1}^{2} a_{2} k-180 a_{0} a_{2}^{2} k+90 a_{2}^{3} k+90 a_{1}^{2} k^{3}+360 a_{0} a_{2} k^{3}-600 a_{2}^{2} k^{3}+1680 a_{2} k^{5}+30 a_{1}^{2} k^{3} p-420 a_{2}^{2} k^{3} p=0, \\
& -225 a_{1} a_{2}^{2} k+450 a_{1} a_{2} k^{3}-120 a_{1} k^{5}+150 a_{1} a_{2} k^{3} p=0, \\
& -90 a_{2}^{3} k+360 a_{2}^{2} k^{3}-720 a_{2} k^{5}+180 a_{2}^{2} k^{3} p=0 .
\end{aligned}
$$

Solving this system with aid of Mathematica we get two solution set 
Set 1:

$$
\begin{aligned}
& m=2\left(-12 k^{5}+10 k^{5} p+5 k^{5} p^{2}-5 k^{3} p \sqrt{k^{4}\left(-4+4 p+p^{2}\right)}\right), \\
& a_{0}=\frac{2}{3}\left(-2 k^{2}-k^{2} p+\sqrt{k^{4}\left(-4+4 p+p^{2}\right)}\right), \\
& a_{1}=0 \\
& a_{2}=2 k^{2}+k^{2} p-\sqrt{-4 k^{4}+4 k^{4} p+k^{4} p^{2}} .
\end{aligned}
$$

Thus using (3.2), (4.3), (4.4) and (4.6) the exact solutions can be found as

$$
\begin{aligned}
& u_{1}(x, t)=\frac{2}{3}\left(-2 k^{2}-k^{2} p+\sqrt{k^{4}\left(-4+4 p+p^{2}\right)}\right) \\
& +\left((42 \pi)^{2}+k^{2} p-\sqrt{-4 k^{4}+4 k^{4} p+k^{4} p^{2}}\right) \tanh \left[k x-\frac{2\left(-12 k^{5}+10 k^{5} p+5 k^{5} p^{2}-5 k^{3} p \sqrt{k^{4}\left(-4+4 p+p^{2}\right)}\right) t^{\alpha}}{\alpha}\right]^{2} .
\end{aligned}
$$

Set 2:

$$
\begin{aligned}
& m=2\left(-12 k^{5}+10 k^{5} p+5 k^{5} p^{2}+5 k^{3} p \sqrt{k^{4}\left(-4+4 p+p^{2}\right)}\right) \\
& a_{0}=\frac{2}{3}\left(-2 k^{2}-k^{2} p-\sqrt{k^{4}\left(-4+4 p+p^{2}\right)}\right) \\
& a_{1}=0 \\
& a_{2}=2 k^{2}+k^{2} p+\sqrt{-4 k^{4}+4 k^{4} p+k^{4} p^{2}}
\end{aligned}
$$

Hence again using (3.2), (4.3), (4.4) and (4.7) the analytical solutions can be found as

$$
\begin{aligned}
& u_{2}(x, t)=\frac{2}{3}\left(-2 k^{2}-k^{2} p-\sqrt{k^{4}\left(-4+4 p+p^{2}\right)}\right) \\
& +\left((42 q)^{2}+k^{2} p+\sqrt{-4 k^{4}+4 k^{4} p+k^{4} p^{2}}\right) \tanh \left[k x-\frac{2\left(-12 k^{5}+10 k^{5} p+5 k^{5} p^{2}+5 k^{3} p \sqrt{k^{4}\left(-4+4 p+p^{2}\right)}\right) t^{\alpha}}{\alpha}\right]^{2} .
\end{aligned}
$$

4.2. Application of Residual Power Series Method. Consider the nonlinear time fractional Kaup-Kupershmidt equation

$$
\frac{\partial^{\alpha} u}{\partial t^{\alpha}}+45 u^{2} \frac{\partial u}{\partial x}-15 p \frac{\partial u}{\partial x} \frac{\partial^{2} u}{\partial x^{2}}-15 u \frac{\partial^{3} u}{\partial x^{3}}+\frac{\partial^{5} u}{\partial x^{5}}=0
$$

with the initial conditions obtained from the exact solution

$$
u(x, 0)=\frac{2}{3}\left(-2 k^{2}-k^{2} p+\sqrt{k^{4}\left(-4+4 p+p^{2}\right)}\right)+\left(2 k^{2}+k^{2} p-\sqrt{-4 k^{4}+4 k^{4} p+k^{4} p^{2}}\right) \tanh [k x]^{2}
$$

The exact solution of time fractional Kaup-Kupersmidt equation is taken as (4.7)

For residual power series

$$
u(x, t)=f(x)+\sum_{n=0}^{\infty} f_{n}(x) \frac{t^{n \alpha}}{\alpha^{n} n !}
$$


and $k$.truncated series of $u(x, t)$

$$
u_{k}(x, t)=f(x)+\sum_{n=0}^{k} f_{n}(x) \frac{t^{n \alpha}}{\alpha^{n} n !}, k=1,2,3, \ldots
$$

The $k_{1}$ th residual function of time fractional Kaup-Kupershmidt equation is:

$$
\operatorname{Resu}_{k}(x, t)=\frac{\partial^{\alpha} u_{k}}{\partial t^{\alpha}}+45 u_{k}^{2} \frac{\partial u_{k}}{\partial x}-15 p \frac{\partial u_{k}}{\partial x} \frac{\partial^{2} u_{k}}{\partial x^{2}}-15 u_{k} \frac{\partial^{3} u_{k}}{\partial x^{3}}+\frac{\partial^{5} u_{k}}{\partial x^{5}}
$$

to determine the coefficient $f_{1}(x)$, in $u_{k}(x, t)$, we should subrogate the 1.th truncated series $u_{1}(x, t)=f(x)+$ $f_{1}(x) \frac{t^{\alpha}}{\alpha}$ into the 1 st truncated residual function

$$
\operatorname{Resu}_{1}(x, t)=\frac{\partial^{\alpha} u_{1}}{\partial t^{\alpha}}+45 u_{1}^{2} \frac{\partial u_{1}}{\partial x}-15 p \frac{\partial u_{1}}{\partial x} \frac{\partial^{2} u_{1}}{\partial x^{2}}-15 u_{1} \frac{\partial^{3} u_{1}}{\partial x^{3}}+\frac{\partial^{5} u_{1}}{\partial x^{5}}
$$

Now for the substitution of $t=0$ through equation $\operatorname{Resu}_{1}(x, t)$ to obtain

$$
\operatorname{Resu}_{1}(x, 0)=f_{1}(x)+45 f^{2}(x) f^{\prime}(x)-15 p f^{\prime}(x) f^{\prime \prime}(x)-15 f(x) f^{(3)}(x)+f^{(5)}(x)
$$

Thus for $\operatorname{Res}_{1}(x, 0)=0$

$$
f_{1}(x)=-45 f^{2}(x) f^{\prime}(x)+15 p f^{\prime}(x) f^{\prime \prime}(x)+15 f(x) f^{(3)}(x)-f^{(5)}(x)
$$

Therefore, we obtain the 1st RPS approximate solution of time-fractional Kaup-Kupershmidt equation as

$$
u_{1}(x, t)=f(x)+\frac{1}{\alpha} t^{\alpha}\left(-45 f^{2}(x) f^{\prime}(x)+15 p f^{\prime}(x) f^{\prime \prime}(x)+15 f(x) f^{(3)}(x)-f^{(5)}(x)\right)
$$

Again, to determine the second unknown coefficient $f_{2}(x)$, we subrogate the $2 n d$ truncated series solution $u_{2}(x, t)=f(x)+f_{1}(x) \frac{t^{\alpha}}{\alpha}+f_{2}(x) \frac{t^{2 \alpha}}{2 \alpha^{2}}$ into the $2 n d$ truncated residual function

$$
\operatorname{Resu}_{2}(x, t)=\frac{\partial^{\alpha} u_{2}}{\partial t^{\alpha}}+45 u_{2}^{2} \frac{\partial u_{2}}{\partial x}-15 p \frac{\partial u_{2}}{\partial x} \frac{\partial^{2} u_{2}}{\partial x^{2}}-15 u_{2} \frac{\partial^{3} u_{2}}{\partial x^{3}}+\frac{\partial^{5} u_{2}}{\partial x^{5}}
$$

to obtain

$$
\begin{aligned}
\operatorname{Resu}_{2}(x, t)= & 45\left(f(x)+\frac{t^{\alpha} f_{1}(x)}{\alpha}+\frac{t^{2 \alpha} f_{2}(x)}{2 \alpha^{2}}\right)\left(f^{\prime}(x)+\frac{t^{\alpha} f_{1}^{\prime}(x)}{\alpha}+\frac{t^{2 \alpha} f_{2}^{\prime}(x)}{2 \alpha^{2}}\right) \\
& -15 p\left(f^{\prime}(x)+\frac{t^{\alpha} f_{1}^{\prime}(x)}{\alpha}+\frac{t^{2 \alpha} f_{2}^{\prime}(x)}{2 \alpha^{2}}\right)\left(f^{\prime \prime}(x)+\frac{t^{\alpha} f_{1}^{\prime \prime}(x)}{\alpha}+\frac{t^{2 \alpha} f_{2}^{\prime \prime}(x)}{2 \alpha^{2}}\right) \\
& -15\left(f(x)+\frac{t^{\alpha} f_{1}(x)}{\alpha}+\frac{t^{2 \alpha} f_{2}(x)}{2 \alpha^{2}}\right)\left(f^{(3)}(x)+\frac{t^{\alpha} f_{1}^{(3)}(x)}{\alpha}+\frac{t^{2 \alpha} f_{2}^{(3)}(x)}{2 \alpha^{2}}\right) \\
+ & f^{(5)}(x)+\frac{t^{\alpha} f_{1}^{(5)}(x)}{\alpha}+\frac{t^{2 \alpha} f_{2}^{(5)}(x)}{2 \alpha^{2}}
\end{aligned}
$$

Now, applying $T_{\alpha}$ on both sides of $\operatorname{Resu}_{2}(x, t)$ and equating to 0 for $t=0$ gives: 


$$
\begin{aligned}
f_{2}(x)= & -90 f(x) f_{1}(x) f^{\prime}(x)-45 f^{2}(x) f_{1}^{\prime}(x)+15 p f_{1}^{\prime}(x) f^{\prime \prime}(x) \\
& +15 p f^{\prime}(x) f_{1}^{\prime \prime}(x)+15 f_{1}(x) f^{(3)}(x)+15 f(x) f_{1}^{(3)}(x)-f_{1}^{(5)}(x)
\end{aligned}
$$

Therefore the $2 n d$ RPS approximate solution of time-fractional Kaup-Kupershmidt is obtained as:

$$
\begin{aligned}
u_{2}(x, t)= & f(x)+\frac{t^{\alpha} f_{1}(x)}{\alpha}+\frac{t^{2 \alpha}}{2 \alpha^{2}}\left(-90 f(x) f_{1}(x) f^{\prime}(x)-45 f^{2}(x) f_{1}^{\prime}(x)+15 p f_{1}^{\prime}(x) f^{\prime \prime}(x)\right. \\
& \left.+15 p f^{\prime}(x) f_{1}^{\prime \prime}(x)+15 f_{1}(x) f^{(3)}(x)+15 f(x) f_{1}^{(3)}(x)-f_{1}^{(5)}(x)\right)
\end{aligned}
$$

In the same manner, we apply the same procedure for $n=3$ to obtain the following results.

$$
\begin{aligned}
& f_{3}(x)=-90 f^{2}(x) f^{\prime}(x)-90 f(x) f_{2}(x) f^{\prime}(x)-180 f(x) f_{1}(x) f_{1}^{\prime}(x)-45 f^{2}(x) f_{2}^{\prime}(x)+15 p f_{2}^{\prime}(x) f^{\prime \prime}(x) \\
& +30 p f_{1}^{\prime}(x) f_{1}^{\prime \prime}(x)+15 p f^{\prime}(x) f_{2}^{\prime \prime}(x)+15 f_{2}(x) f^{(3)}(x)+30 f_{1}(x) f_{1}^{(3)}(x)+f(x) f_{2}^{(3)}(x)-f_{2}^{(5)}(x) \\
& u_{3}(x, t)=\quad f(x)+\frac{t^{\alpha} f_{1}(x)}{\alpha}+\frac{t^{2 \alpha} f_{2}(x)}{2 \alpha^{2}}+\frac{t^{3 \alpha}}{6 \alpha^{3}}\left(-90 f^{2}(x) f^{\prime}(x)-90 f(x) f_{2}(x) f^{\prime}(x)\right. \\
& -180 f(x) f_{1}(x) f_{1}^{\prime}(x)-45 f^{2}(x) f_{2}^{\prime}(x)+15 p f_{2}^{\prime}(x) f^{\prime \prime}(x)+30 p f_{1}^{\prime}(x) f_{1}^{\prime \prime}(x) \\
& \left.+15 p f^{\prime}(x) f_{2}^{\prime \prime}(x)+15 f_{2}(x) f^{(3)}(x)+30 f_{1}(x) f_{1}^{(3)}(x)+f(x) f_{2}^{(3)}(x)-f_{2}^{(5)}(x)\right)
\end{aligned}
$$

\begin{tabular}{ccccccc}
\hline \multicolumn{3}{c}{$\alpha=0.25$} & $\alpha=0.50$ & $\alpha=0.75$ & \multicolumn{3}{c}{$\alpha=1$} \\
\hline$x$ & $u_{3}$ & $u_{3}$ & $u_{3}$ & RPSM & Exact & Absolute error \\
0 & -0.165431 & -0.166569 & -0.166653 & -0.166664 & -0.166664 & $1.58945 \mathrm{E}-11$ \\
0.1 & -0.16656 & -0.166438 & -0.166214 & -0.166118 & -0.166118 & $1.55637 \mathrm{E}-11$ \\
0.2 & -0.166442 & -0.165064 & -0.164536 & -0.164335 & -0.164335 & $1.45830 \mathrm{E}-11$ \\
0.3 & -0.165082 & -0.162474 & -0.161652 & -0.161350 & -0.161350 & $1.30444 \mathrm{E}-11$ \\
0.4 & -0.162504 & -0.158718 & -0.157618 & -0.157222 & -0.157222 & $1.10004 \mathrm{E}-11$ \\
0.5 & -0.158761 & -0.153869 & -0.152513 & -0.152028 & -0.152028 & $8.67079 \mathrm{E}-12$ \\
0.6 & -0.153924 & -0.148018 & -0.146429 & -0.145866 & -0.145866 & $6.11641 \mathrm{E}-12$ \\
0.7 & -0.148083 & -0.141269 & -0.139477 & -0.138844 & -0.138844 & $3.52712 \mathrm{E}-12$ \\
0.8 & -0.141344 & -0.133740 & -0.131774 & -0.131083 & -0.131083 & $1.02934 \mathrm{E}-12$ \\
0.9 & -0.133824 & -0.125555 & -0.123446 & -0.122708 & -0.122708 & $1.25487 \mathrm{E}-12$ \\
1.0 & -0.125646 & -0.116841 & -0.114621 & -0.113846 & -0.113846 & $3.22763 \mathrm{E}-12$ \\
\hline
\end{tabular}

TABLE 1. Comparison of numerical results for $\lambda=0.1, \omega=1, \mu=0, t=0.1, p=2.5$ and $k=0.5$ with different values of $\alpha$.

\section{Conclusion And Discussion}

In this paper, exact and approximate solutions of the nonlinear time-fractional Kaup-Kupershmidt differential equation with the tanh method and residual power series method (RPSM) are obtained. With the help of conformable fractional derivative definition we can easily transform fractional differential equations to the known classical differential equations. By these methods and conformable fractional derivative definition, it is shown that there is no need another complex method and complex definition. Approximate and exact solutions of timefractional Kaup-Kupershmidt differential equation are compered. Absolute errors are given with approximate 


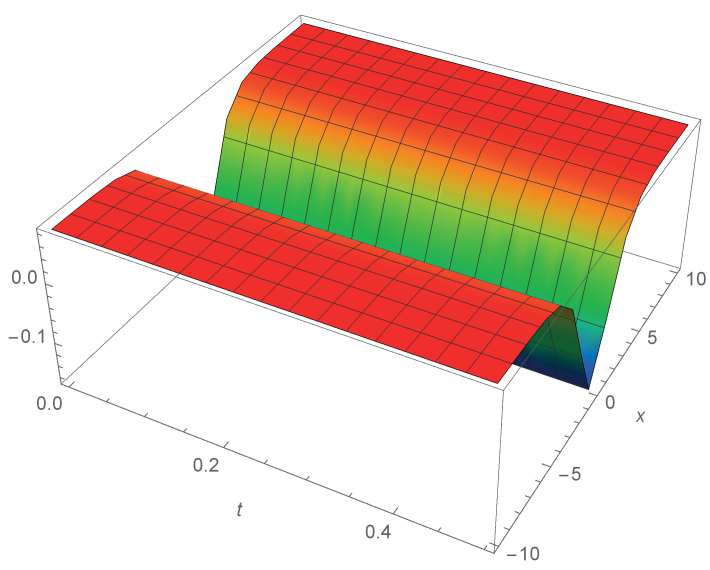

(A) RPSM solution for $\alpha=1$.

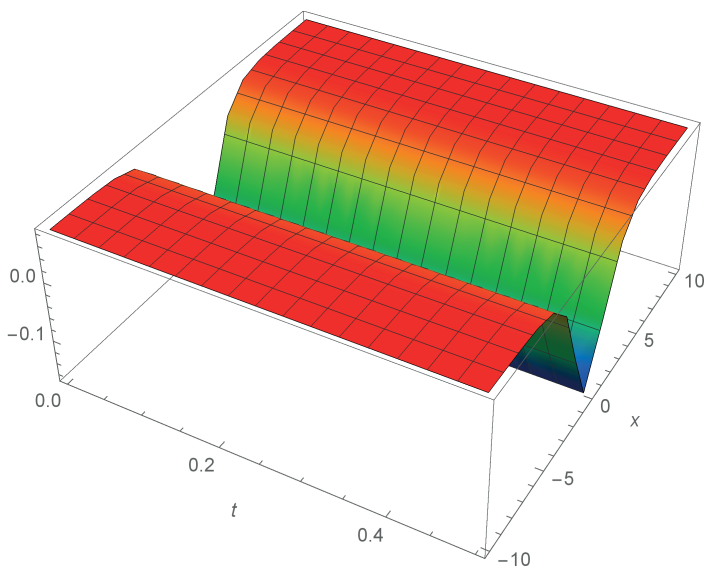

(в) Exact solution for $\alpha=1$

FIGURE 1

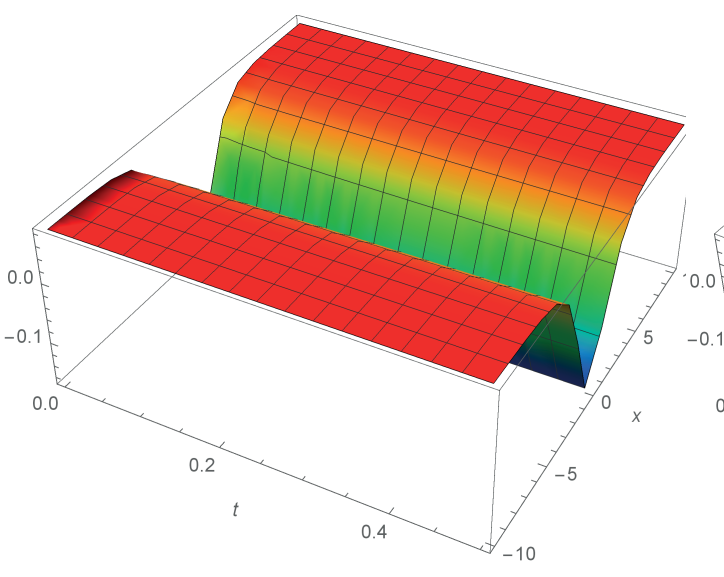

(A) RPSM solution for $\alpha=0.25$.

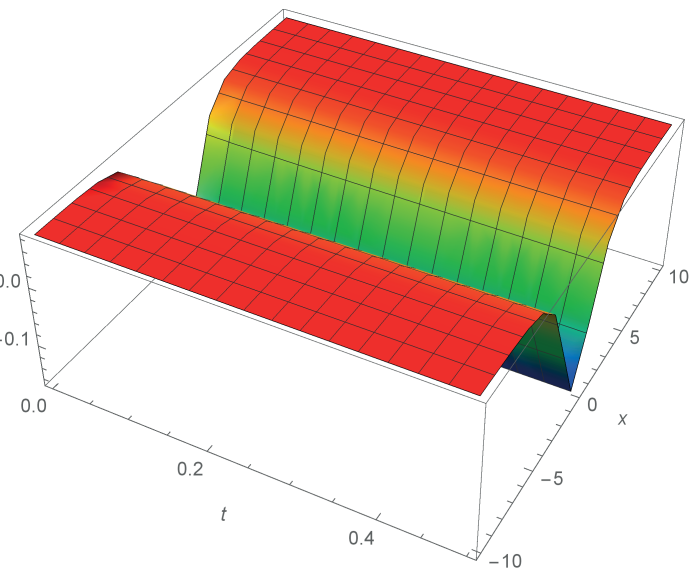

(в) RPSM solution for $\alpha=0.50$

FigURE 2

and exact solutions with the help of graphs and tables. Also, it is seen that conformable fractional derivative is clearer, simpler and understandable than other fractional derivative definitions. 


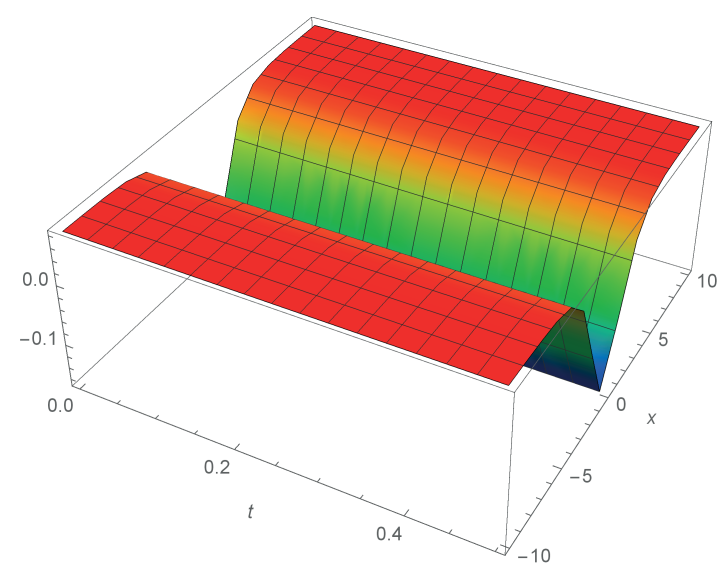

FIGURE 3. RPSM solution for $\alpha=0.75$.

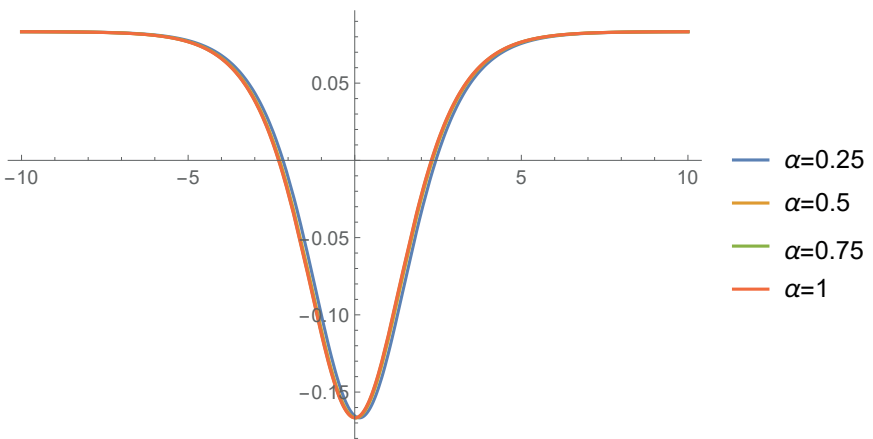

Figure 4. Plots of $u_{3}(x, t)$ versus space $x$ for different values of $\alpha$ at $t=0.1$..

\section{REFERENCES}

[1] Abdeljawad, T, On conformable fractional calculus. J. Comput. Appl. Math. 279 (2015), 57-66.

[2] Ahmad, R. S. An analytical solution of the fractional Navier-Stokes equation by residual power series method., Zarqa University, Doctoral dissertation, 10-90, 2015.

[3] Alabsi T.Y., Solution of Conformable Fractional Navier-Stokes Equation, M.S. thesis, Zarqa University, 2017.

[4] Alquran, M. Analytical solutions of fractional foam drainage equation by residual power series method, Math. Sci. 8 (4) (2014), 153-160.

[5] Arikoglu, A., \& Ozkol, I. Solution of fractional differential equations by using differential transform method. Chaos Solitons Fractals, 34 (5) (2007), 1473-1481.

[6] Atangana, Abdon, Dumitru Baleanu, and Ahmed Alsaedi. New properties of conformable derivative. Open Math. 13 (2015), 889-898.

[7] Caputo, M., Linear models of dissipation whose Q is almost frequency independent-II, Geophys. J. Int. 13 (5) (1967), $529-539$.

[8] Cenesiz Y., Kurt A. and Tasbozan O. On the New Solutions of the Conformable Time Fractional Generalized Hirota-Satsuma Coupled KdV System. Ann. West Univ. Timisoara, Math. Comput. Sci. 55 (2017), 37-50.

[9] Das, S., Functional fractional calculus, Springer, 2011.

[10] Diethelm, K., The analysis of fractional differential equations, Springer, 2010

[11] El-Ajou, A., Arqub, O. A., Zhour, Z. A., \& Momani, S. New results on fractional power series: theories and applications, Entropy, 15(12) (2013), 5305-5323.

[12] Jaradat, H. M., Al-Shara, S., Khan, Q. J., Alquran, M., \& Al-Khaled, K. Analytical solution of time-fractional Drinfeld-Sokolov-Wilson system using residual power series method, IAENG International J. Appl. Math. 46 (2016), 64-70.

[13] Kexue, L., \& Jigen, P. Laplace transform and fractional differential equations. Appl. Math. Lett. 24 (12) (2011), $2019-2023$.

[14] Khalil, Roshdi, et al. A new definition of fractional derivative. J. Comput. Appl. Math. 264 (2014), 65-70. 
[15] Kumar, A., Kumar, S., \& Singh, M., Residual power series method for fractional Sharma-Tasso-Olever equation, Commun. Numer. Anal. 2016 (2016), Article ID cna-00235.

[16] Kurt, A., O. Tasbozan, and D. Baleanu. New solutions for conformable fractional Nizhnik-Novikov-Veselov system via $G / / G$ expansion method and homotopy analysis methods. Opt. Quant. Electr. 49 (2017), 333.

[17] W. Malfliet, The tanh method: a tool for solving certain classes of non-linear PDEs, Math. Methods Appl. Sci. 28 (2005), $2031-2035$.

[18] W. Malfliet, The tanh method: a tool for solving certain classes of nonlinear evolution and wave equations, J. Comput. Appl. Math. 164 (2004), 529-541.

[19] Ray, S. S., \& Bera, R. K. An approximate solution of a nonlinear fractional differential equation by Adomian decomposition method. Appl. Math. Comput. 167(2005), 561-571.

[20] Şenol, M., \& Dolapci, I. T. On the Perturbation-Iteration Algorithm for fractional differential equations, J. King Saud Univ., Sci. 28(2016), 69-74.

[21] Wazwaz, A.M., The tanh method for traveling wave solutions of nonlinear equations. Appl. Math. Comput. 154(2004), 713-723.

[22] Yildirim, A. An algorithm for solving the fractional nonlinear Schrödinger equation by means of the homotopy perturbation method. Int. J. Nonlinear Sci. Numer. Simul. 10 (4) (2009), 445-450. 